\title{
Exposure or cognitive restructuring were more effective than relaxation in treating post-traumatic stress disorder
}

\author{
Marks I, Lovell K, Noshirvani H, et al. Treatment of posttraumatic stress disorder by exposure and/or cognitive restructuring. A \\ controlled study. Arch Gen Psychiatry 1998 Apr;55:31 7-25.
}

\section{Question}

In patients with post-traumatic stress disorder (PTSD), what are the effects of prolonged exposure and cognitive restructuring, alone and in combination, compared with relaxation training?

Design

Randomised controlled trial with 6 months follow up.

Setting

London, UK.

\section{Patients}

87 outpatients who had PTSD according to DSM-III-R criteria for $\geqslant 6$ months and were $16-65$ years of age (mean age 38 y, $64 \%$ men). Exclusion criteria were melancholia or suicidal intent, organic brain disease, past or present psychosis, treatment with an antidepressant drug unless the patient had been receiving a stable dose for $\geqslant 3$ months, receipt of diazepam at a dose of $\geqslant 10$ $\mathrm{mg} /$ day, ingestion of $\geqslant 30$ alcohol units/week, or past exposure or cognitive therapy for PTSD. 77 patients (89\%) completed the 10 session intervention.

\section{Intervention}

Patients were stratified for personal or impersonal trauma and allocated to exposure therapy $(n=23)$, cognitive restructuring $(\mathrm{n}=19)$, exposure combined with cognitive restructuring $(n=24)$, or relaxation $(n=21)$. Exposure therapy involved imaginal and prolonged live exposure; cognitive restructuring involved identifying and monitoring negative automatic thoughts and evaluating them with probabilistic reasoning and Socratic questioning; combined therapy involved imaginal exposure and cognitive restructuring for the first 5 sessions with live exposure and cognitive restructuring for the latter 5 sessions; and relaxation involved learning relaxation techniques.

\begin{abstract}
Main outcome measures
Change in score on 12 primary measures: ClinicianAdministered PTSD Scale (CAPS 2) total of 3 clusters and severity, Impact of Events Scale (IES) (self rated), Beck Depression Inventory (self rated), Global Improvement scale (self and assessor rated), main problem (self and assessor rated), total of 4 goals to deal with the problem (self and assessor rated), and Work and Social Adjustment scale (self and assessor rated).
\end{abstract}

\section{Main results}

Using analysis of covariance, improvement was greater in patients who received exposure, cognitive, or combined therapy than patients who received relaxation therapy for 11 of the 12 primary measures at post-treatment (week 11), for 6 primary measures at 1 month of follow up, and for 8 primary measures at 3 months of follow up. The effect sizes (mean of the change score divided by the standard deviation of the change score; $\geqslant 1$ was considered clinically meaningful) were 1 to 2.5 from posttreatment onward for all primary measures except for self assessed work and social adjustment which was 0.90 at post-treatment and 1 month of follow up, and were mostly greater than the effect sizes for relaxation. At post-treatment, the percentage of patients improved was $60 \%, 50 \%$, and $58 \%$ for the exposure, cognitive, and combined therapy groups, respectively, compared with $20 \%$ in the relaxation group $(\mathrm{p}=0.04)$ on the IES.

\section{Conclusion}

Exposure therapy and cognitive restructuring, alone or combined, were more effective than relaxation in patients with posttraumatic stress disorder.

Source of funding: Wellcome Trust, London, UK.

For correspondence: Dr I Marks, Institute of Psychiatry, London SE5 8AF, UK. Fax +44 (0)171 740 5244.

\section{Commentary}

The effectiveness of various cognitive behavioural psychotherapeutic interventions generally has been shown by comparing them with pharmacotherapy or with some control condition that involves no active treatment (eg, being placed on a waiting list). The study by Marks et al is 1 of the first to directly compare 3 different therapies which address the 3 major symptom complexes of PTSD. Prolonged exposure therapy targets the avoidance of trauma related cues, cognitive restructuring focuses on negative automatic thoughts, and relaxation focuses on symptoms of arousal. All 3 treatments are somewhat effective because they enable patients to control their symptoms.

The results of this study, however, raise 2 important questions: (1) which therapy is most effective? And (2) is it better to combine therapies? Exposure therapy and cognitive restructuring appear to be equally effective in treating symptoms of PTSD compared with relaxation. When compared with each other, however, the results are mixed and somewhat confusing because of the multiple comparisons. Although the study found the 2 therapies to be equivalent, an examination of the effect sizes suggests that cognitive restructuring is more effective in the short term (post-treatment and 1 mo follow up), and exposure therapy is more effective in the long term (3 and 6 mo follow up). Each therapy also seems to be more effective with a specific set of symptoms. Hence, exposure therapy is more effective in reducing the symptoms that predominate in the IES, whereas cognitive restructuring is more effective in reducing the symptoms that predominate in the CAPS 2. The finding that the 2 therapies used in combination are no more effective than each therapy used alone may say as much about how re-experiencing, avoidance, and arousal are related to one another as it does about the difficulty of implementing more than 1 therapeutic modality at a time. It also shows the fallacy of assuming an equivalence between a multimodal approach that combines psychotherapy with pharmacotherapy and an approach that combines more than 1 form of psychotherapy.

Nevertheless, clinicians should seriously consider using a cognitive behavioural intervention in combination with pharmacotherapy in treating PTSD. The choice of therapy should be determined by which of the 3 symptom complexes creates the most impairment for the patient and whether the therapy serves short or long term objectives.

Lawrence A Palinkas, PhD University of California, San Diego San Diego, California, USA 